\section{De macro à micro : I'histoire de la plaquette}

Najet Debili, William Vainchenker
Inserm UMR 790, université Paris XI,

Institut Gustave Roussy,

39, rue Camille Desmoulins,

94805 Villejuif, France.

verpre@igr.fr

najet.debili@igr.fr

qui s'invagine dans le cytoplasme du mégacaryocyte. Déjà en 1956, J.P. Thierry et $M$. Bessis observaient qu'in vitro des mégacaryocytes étaient capables de prendre l'aspect de pieuvres en émettant de nombreux pseudopodes [2], et J.M. Radley et son équipe ont identifié dans la moelle ces longs pseudopodes, traversant la barrière endothéliale, appelés proplaquettes [3]. Ces résultats ont été confirmés ensuite par plusieurs autres équipes, mais ils se fondent sur l'observation d'un nombre limité de mégacaryocytes.

La découverte de la thrombopoïétine a changé la donne en facilitant l'approche expérimentale de ces processus, car l'obtention d'un grand nombre de mégacaryocytes in vitro à partir de progéniteurs mégacaryocytaires est maintenant possible. En culture, les mégacaryocytes forment spontanément des proplaquettes avant de libérer de vraies plaquettes, et les données initiales de J.M. Radley ont été confirmées par l'observation que les proplaquettes correspondent à des déroulements des membranes de démarcation [4-6]. Les microtubules en glissant les uns sur les autres procurent la force de protrusion [5-8] et régulent le trafic des organites comme les mitochondries et les granules, du corps du mégacaryocyte vers les proplaquettes [9]. Autre argument confirmant le rôle des proplaquettes dans la formation des plaquettes, l'invalidation du facteur de transcription NF-E2 entraîne une thrombopénie profonde parce que les mégacaryocytes sont incapables de former des proplaquettes [10]. Or NF- $\varepsilon 2$ contrôle au niveau transcriptionnel l'expression de la 
tubuline $\beta 1$, principale tubuline du mégacaryocyte mature. L'équipe d'Italiano et al. a montré qu'au bout des proplaquettes, les microtubules s'enroulaient pour «préformer» une plaquette qui était ensuite libérée à l'extrémité de la proplaquette [6]. Les plaquettes peuvent naître différemment: d'autres équipes ont ainsi montré que les proplaquettes se cassaient en culture au niveau de zones rétrécies et se détachaient du mégacaryocyte pour se fragmenter secondairement [5] (Figure 1). Mais une des limites de ces descriptions de la «naissance» des plaquettes est que les observations se faisaient in vitro dans des systèmes de culture très artificiels.

\section{Le film de la naissance \\ d'une pro-plaquette in vivo}

Ce qui fait tout l'interêt du travail de T. Junt et al., dont les résultats ont été récemment publiés dans Science, c'est la démonstration que ces auteurs font, par une approche très innovante technologiquement, de la production plaquettaire in vivo, qui est bien liée à la formation de proplaquettes dans les sinusoïdes de la moelle [11]. Grâce à l'utilisation d'un microscope biphotonique permettant une visualisation par microscopie intravitale, ils ont pu observer des mégacaryocytes formant des proplaquettes in vivo chez des souris ayant des mégacaryocytes spontanément fluorescents, par knock in du gène codant la GPIIb plaquettaire (CD41) par la EyFP (extended yellow fluorescent protein). Les auteurs montrent que les mégacaryocytes étendent les proplaquettes dans la circulation vasculaire et que ces proplaquettes se cassent dans les sinusoïdes sous l'effet du flux sanguin, en libérant parfois des fragments de très grande taille qui ensuite se fragmentent à nouveau dans la circulation. La formation directe de plaquettes, sans passer par une étape intermédiaire de proplaquettes, semble plus rare, mais elle est techniquement difficile à visualiser. Ces résultats confirment ceux de 0 . Behnke qui avait identifié dans le sang circulant chez l'homme, la présence de proplaquettes capables de se fragmenter in vitro en plaquettes [12]. T. Junt et al. ont également observé la migration d'un mégacaryocyte qui, momentanément, obstrue la circulation dans les sinusoïdes.

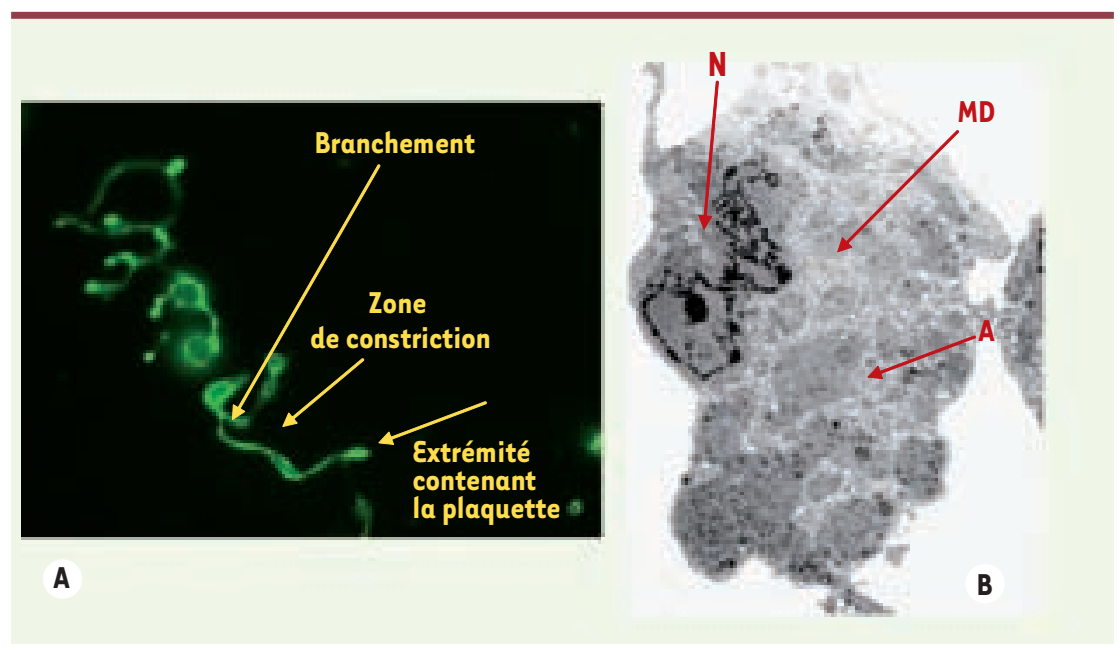

Figure 1. Structure mégacaryocytaire et formation de proplaquettes. A. Formation de proplaquettes à partir de mégacaryocytes obtenus en culture via la différenciation de cellules progénitrices immatures $C D 34^{+}$de sang humain : les cellules sont déposées sur des lames recouvertes de polylysine, fixées, perméabilisées puis immuo-marquées avec un anticorps dirigé contre la tubuline. $B$. Ultrastructure d'un mégacaryocyte typique obtenu in vitro: le mégacaryocyte est de grande taille, présente un noyau polylobulé ( $N$ ), des membranes de démarcation bien développées (MD) ainsi que de nombreux granules alpha $(A)$.

L'une des observations les plus curieuses de ce travail concerne la présence d'extensions, et peut être la présence de fragments de cytoplasme, voire de plaquettes, dans l'insterstitium de la moelle. Or, il est fondamental que la plaquettogenèse ait lieu dans la circulation et non à l'intérieur de la moelle, car les plaquettes n'ont pas la propriété de traverser la barrière endothéliale. Il est donc possible que ces fragments détectés dans l'environnement médullaire ne soient pas de vraies plaquettes, mais représentent des particules qui assurent la régulation du stroma médullaire. On pourrait spéculer sur le rôle de ce processus en pathologie: par exemple, l'augmentation de ces fragments interstitiels pourrait faciliter le développement d'une myélofibrose, via une sécrétion excessive de TGF- $\beta 1$ (dont les mégacaryocytes sont un réservoir important) dans l'environnement médullaire.

\section{Mégacaryocyte et cellules}

\section{endothéliales : ça passe ou ça casse}

Le déroulement d'une plaquettogenèse efficace exige que le mégacaryocyte mature migre d'un environnement ostéoblastique vers les sinusoïdes. II existe des arguments impliquant la chimiokine SDF-1/CCL12 dans ce processus de chimio-attraction, bien que des résultats très contradictoires aient été rapportés et que des souris receveuses ayant reçu une greffe de moelle osseuse de souris donneuses $C X C R 4^{-/-}$ne soient pas thrombopéniques [13]. On ne sait pas si une molécule régule la formation de proplaquettes, mais en tous les cas la thrombopoïétine n'est pas directement impliquée dans ce processus, expliquant que son effet sur la production plaquettaire soit retardé. II existe des arguments in vitro pour penser que l'environnement intra-médullaire, en particulier le collagène, inhibe la formation des proplaquettes [14]. En revanche, lorsque le mégacaryocyte migre à proximité des cellules endothéliales il entre en contact avec un autre environnement 
contenant du facteur von Willebrand et du fibrinogène, deux facteurs capables d'induire la formation des proplaquettes [15]. La migration du mégacaryocyte dans un environnement approprié serait donc le mécanisme régulateur de la formation de proplaquettes, et le flux sanguin contrôlerait la libération des plaquettes par cassure des proplaquettes. On peut penser que des altérations de ce mécanisme de régulation par le stroma médullaire pourrait être à l'origine de thrombopénies [16]. De macro à micro : beaucoup reste à découvrir. $\diamond$ From giant to micro: a platelet story

\section{RéFÉRENCES}

1. Zucker-Franklin D, Petursson S. Thrombocytopoiesis: analysis by membrane tracer and freeze-fracture studies on fresh human and cultured mouse megakaryocytes. J Cell Biol 1984 ; 99 : 390-402.

\section{DICTIONNAIRE DE MEDECINE FLAMMARION}

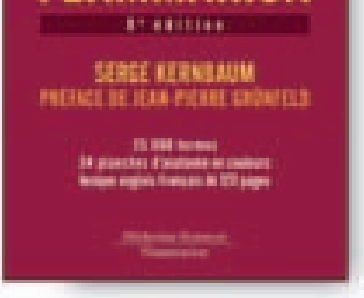

Avril 2008 - 1133 paces Prix publle Tre : 30 e

\section{- Vbio la 7 dolion din gand chassique, reconvu pour sa fabilith. - (Panorama du Medecini)} - Le dictionnaive de medecine farmavion est remarquable. - (La Presse Medical)
2. Thiery JP, Bessis M. Mécanisme de la plaquettogenèse. Rev Hematol 1956 ; 11: 162-74.

3. Radley JM, Haller CJ. The demarcation membrane system of the megakaryocyte: a misnomer? Blood $1982 ; 60: 213-9$.

4. Choi ES, Nichol JL, Hokom MM, et al. Platelets generated in vitro from proplatelet-displaying human megakaryocytes are functional. Blood 1995; $85: 402-13$.

5. Cramer $\varepsilon M$, Norol F, Guichard J, et al. Ultrastructure of platelet formation by human megakaryocytes cultured with the Mpl ligand. Blood 1997 ; 89: 2336-46.

6. Italiano JE Jr, Lecine P, Shivdasani RA, Hartwig JH. Blood platelets are assembled principally at the ends of proplatelet processes produced by differentiated megakaryocytes. J Cell Biol 1999; 147 : 1299-312.

7. Tablin F, Castro M, Leven RM. Blood platelet formation in vitro. The role of the cytoskeleton in megakaryocyte fragmentation. J Cell Sci $1990 ; 97:$ 59-70.

8. Patel SR, Richardson JL, Schulze H, et al. Differential roles of microtubule assembly and sliding in proplatelet formation by megakaryocytes. Blood 2005; 106 : 4076-85.

9. Richardson JL, Shivdasani RA, Boers C, et al. Mechanisms of organelle transport and capture along proplatelets during platelet production. Blood 2005; $106: 4066-75$.
10. Lecine $P$, Villeval JL, Vyas $P$, et al. Mice lacking transcription factor NF- $\varepsilon 2$ provide in vivo validation show a platelet production defect that is intrinsic to megakaryocytes. Blood 1998; 92 : 1608-16.

11. Junt $T$, Schulze $H$, Chen $Z$, et al. Dynamic visualization of thrombopoiesis within bone marrow. Science 2007 317: 1767-70.

12. Behnke 0, Forer A. From megakaryocytes to platelets: platelet morphogenesis takes place in the bloodstream. EurJ Haematol 1998; 61 (suppl) : 3-23.

13. Foudi $A$, Jarrier $P$, Zhang $Y$, et al. Reduced retention of radioprotective hematopoietic cells within the bone marrow microenvironment in $\mathrm{CXCR4^{-/- }}$ chimeric mice. Blood 2006; 107 : 2243-51.

14. Sabri S, Jandrot-Perrus M, Bertoglio J, et al. Differential regulation of actin stress fiber assembly and proplatelet formation by alpha2betal integrin and GPVI in human megakaryocytes. Blood 2004 ; $104: 3117-25$.

15. Larson MK, Watson SP. Regulation of proplatelet formation and platelet release by integrin alpha llb beta3. Blood 2006; 108 : 1509-14

16. Sabri S, Foudi A, Boukour S, et al. Deficiency in the Wiskott-Aldrich protein induces premature proplatelet formation and platelet production in the bone marrow compartment. Blood 2006; $108: 134-40$. of the proplatelet model of thrombocytopoiesis and

\section{La 8* edition d'un grand classique : Dictionnaire de Médecine Flammarion} Serge KERNBAUM

Plus de 1000 nouvelles entries, des definitions reactualisees, de nombreus schbmas danatomie en couleurs, sur 32 planches horstexte. Au total, plus de 23000 termes, avec leurs definitions, leur Gquivalent andais et, quand c'est justifié. un developpement clinique ou para clinique.

+ Un lexique andais/trancais de plus de 100 pages, des abrewations courantes en biologe et medecine, des constantes biologiques, des unites de mesures, etr.

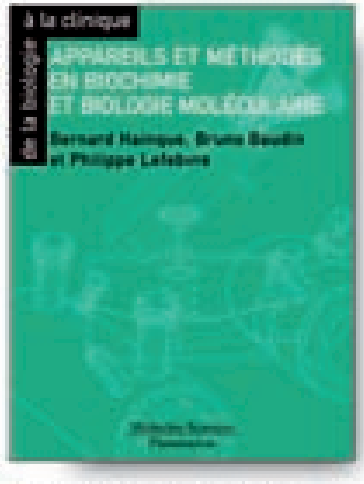

Avril 2009 - 454 paces

200 iffustrations

Pris public Tre : 756

\section{Appareils et méthodes} en biochimie et biologie moléculaire (z édition) Bemard HAINQUE, Bruno BAUDIN et Philippe LEFEBVRE

\section{+ Très nichement illusitre. \\ + Expose de facon complete et pratique toutas

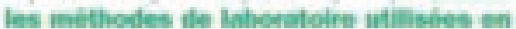 blochimie et bloiogle molèculatire: \\ - les unites, l'instrumentation, la prépara tion des réactifs et des echantillons bio logiques, les mbthodes d'extraction et de fractionnement. toutes les méthodes soectroscopigues, eraymatiques, etc.: \\ - leurs principes, leurs outis, le clonage de rADN. les puses a ADN. les interactions ligands recepteurs et acides nucleiques: proteines.}

Dans fous les chaplines, sont decrits les outils, les méthodes. leurs ressuttats, faisant de ce Ihre un eutil indispensable.

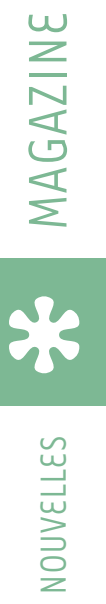

\section{En vente chez vote libraire speciolise, por correspondance ou sur natre site wmw medecine flommorion com

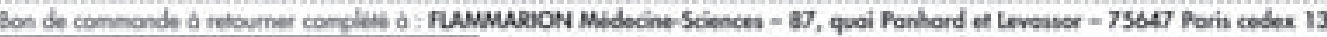

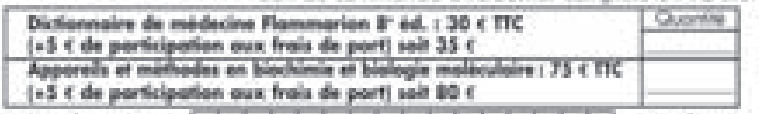

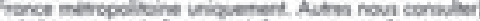
a ieins men reglement a la cemmande : Moriout kad de

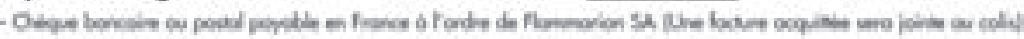

